\title{
THE NONREALIZABILITY OF MODULAR RINGS OF POLYNOMIAL INVARIANTS BY THE COHOMOLOGY OF A TOPOLOGICAL SPACE
}

\author{
LARRY SMITH
}

\begin{abstract}
Let $G<\mathrm{GL}\left(n ; \mathbf{F}_{p}\right)$ be a $p$-group, $p$ an odd prime, and $R^{*}:=\mathbf{F}_{p}\left[x_{1}, \ldots, x_{n}\right]^{G}$ the ring of invariants. The purpose of this note is to prove that in the case where $R^{*}$ is a graded polynomial algebra, where $\operatorname{deg} x_{1}=\cdots=$ $\operatorname{deg} x_{n}=2$, then there is no space $X$ such that $H^{*}\left(X ; \mathbf{F}_{p}\right) \simeq R^{*}$. This complements the work of Clark and Ewing [3] and Adams and Wilkerson [1] on the case $p \nmid[G ; 1]$.
\end{abstract}

The purpose of this note is to record a sort of complement to the theorems of Clark and Ewing [3] and Adams and Wilkerson [1]. In the preceding cited articles it is shown that every polynomial algebra over the Galois field $\mathbf{F}_{p}$, generated by elements of degrees relatively prime to $p$, which is an unstable algebra over the $\bmod p$ Steenrod algebra, arises as the ring of invariants of a finite group of order prime to $p$ which is generated by pseudoreflections, and is realized as the cohomology algebra of at least one topological space. For $p$-groups whose rings of invariants are polynomial the situation is quite different. We have

Proposition. Let $p$ be an odd prime and $G<\mathrm{GL}\left(n ; \mathbf{F}_{p}\right)$ be a p-group. Suppose that the ring of invariants $R^{*}:=\mathbf{F}_{p}\left[x_{1}, \ldots, x_{n}\right]^{G}$ is a polynomial algebra, where $x_{1}, \ldots, x_{n}$ all have degree 2 . Then $R^{*}$ cannot arise as the $\mathbf{F}_{p}$ cohomology of a space.

REMARKS. (1) Since $x_{1}, \ldots, x_{n}$ all have degree 2 there is a unique structure of unstable algebra over the Steenrod algebra on $\mathbf{F}_{p}\left[x_{1}, \ldots, x_{n}\right]$, which in turn induces an analogous structure on $R^{*}$.

(2) There are many examples of $p$-groups satisfying the hypothesis of the proposition, for example, the upper triangular matrices in $\operatorname{GL}\left(n ; \mathbf{F}_{p}\right)$. For $n=2$ the corresponding ring of invariants is $\mathbf{F}_{p}\left[\rho_{1}, \rho_{2}\right]$ where one may choose $\rho_{1}=x_{1}$, $\rho_{2}=x_{2}^{p}-x_{2}^{p-1} x_{1}$. The action of the Steenrod algebra is determined by unstability and the equation $P^{1} \rho_{2}=\rho_{1}^{p-1} \rho_{2}$.

(3) Recently, Nakajima [5] has characterized the $p$-groups $G<\mathrm{GL}\left(n ; \mathbf{F}_{p}\right)$ with a polynomial ring as invariants.

Proof of Proposition. Write $R^{*}=\mathbf{F}_{p}\left[\rho_{1}, \ldots, \rho_{n}\right]$ where $\operatorname{deg} \rho_{1} \leqslant \cdots \leqslant \operatorname{deg} \rho_{n}$. Let $\rho_{1}, \ldots, \rho_{k}$ be the generators of degree 2. (There is always at least one such. To see this set $V:=\bigoplus_{n} \mathbf{F}_{p}$ and note that $V-V^{G}$ is a union of orbits consisting of more than one point in each orbit. Since $G$ is a $p$-group this means $\left|V-V^{G}\right| \equiv 0(p)$

Received by the editors October 21, 1981.

1980 Mathematics Subject Classification. Primary 55P99; Secondary 55P45, 55R35.

(C)1982 American Mathematical Society 0002-9939/82/0000-0239/\$01.25 
where || denotes the cardinality of the underlying set. But $|V|=p^{n}$ and thus $\left|V^{G}\right| \equiv 0(p)$, so $V^{G} \neq\{0\}$.) The elements $\rho_{1}, \ldots, \rho_{k}$ are then a basis for $V^{G}$. Suppose $X$ is a topological space such that $H^{*}(X ; \mathbf{F}) \simeq R^{*}$. then $\rho_{1}, \ldots, \rho_{\mathrm{k}}$ is the reduction of integral classes, so there is a map

$$
f: X \rightarrow \times_{k} \mathbf{C P}(\infty)
$$

inducing an isomorphism on $H^{2}\left(; \mathbf{F}_{p}\right)$. Let $Y$ be the fiber of $f$. An easy spectral sequence argument shows (eg. $[6,3.1])$ that

$$
H^{*}\left(Y ; \mathbf{F}_{p}\right) \simeq \mathbf{F}_{p}\left[\rho_{k+1}, \ldots, \rho_{n}\right]
$$

From [2, Example 8, p. 139] it follows that

$$
p^{s}=|G|=\prod_{i=1}^{n-k}\left(\frac{\operatorname{deg} \rho_{k+i}}{2}\right)
$$

and thus $\frac{1}{2} \operatorname{deg} \rho_{k+i}$ is a power of $p$ for $i=1, \ldots, n-k$. Suppose $\operatorname{deg} \rho_{k+1}=2 p^{r+1}$, $r \geqslant 0$. Then by [4]

$$
\rho_{k+1}^{p}=P^{p^{r+1}} \rho_{k+1}=\sum_{k=1}^{r} a_{k_{i}} \Psi_{i}\left(\rho_{k+1}\right)+b_{k} \Re\left(\rho_{k+1}\right)
$$

(the $\Gamma_{j}$ terms not figuring in the formula since they are of odd degree), where $\operatorname{deg} \Re=4(p-1)$ and $\operatorname{deg} \Psi_{i}=2 p^{i}(p-1)$. So

$$
\begin{aligned}
& \operatorname{deg} \Psi_{i}\left(\rho_{k+1}\right)=2 p^{r+1}+2 p^{i}(p-1) \neq 0 \bmod 2 p^{r}, \\
& \operatorname{deg} \Re\left(\rho_{k+1}\right)=2 p^{r+1}+4(p-1) \neq 0 \bmod 2 p^{r} .
\end{aligned}
$$

But $H^{i}\left(Y ; \mathbf{F}_{p}\right)=0: 2 p^{r+1} \leqslant i \leqslant 2 p^{r+2}$ and $i \neq 0 \bmod 2 p^{r+1}$. Whence from (*) we get $\rho_{k+1}^{p}=0$ which is a contradiction.

\section{REFERENCES}

1. J. F. Adams and C. W. Wilkerson, Finite H-spaces and algebras over the Steenrod algebra, Ann. of Math. (2) 111 (1980), 95-143; correction, Ann. of Math. (2) 113 (1981), 621-622.

2. N. Bourbaki, Groupes et algèbres de Lie, Chapitre 5, Hermann, Paris, 1968.

3. A. Clark and J. Ewing, The realization of polynomial algebras as cohomology rings, Pacific J. Math. 50 (1974), 425-434.

4. A. Liulevicius, The factorization of cyclic reduced powers by secondary cohomology operations, Mem. Amer. Math. Soc., No. 42 (1962).

5. H. Nakajima, Modular representations of p-groups with regular rings of invariants, Proc. Japan Acad. 58 (1980), 469-473.

6. L. Smith, Homological algebra and the Eilenberg-Moore spectral sequence, Trans. Amer. Math. Soc. 129 (1967), 58-93.

Mathematisches Institut, Georg Augustus Universitä, D 3400 Gottingen, West Germany 